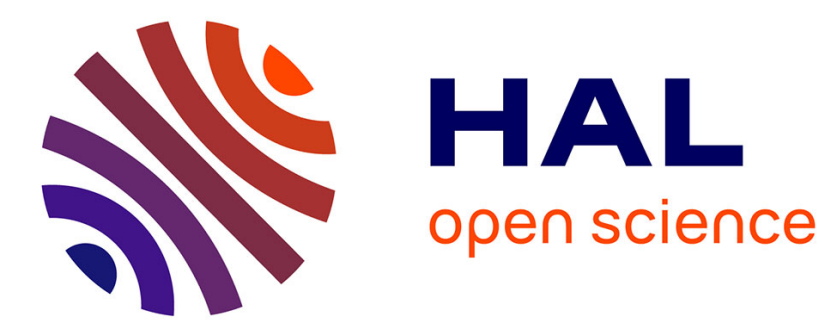

\title{
Accounting for the variability of rock detachment conditions in designing rockfall protection structures
}

Franck Bourrier, Julien Baroth, Stéphane Lambert

\section{To cite this version:}

Franck Bourrier, Julien Baroth, Stéphane Lambert. Accounting for the variability of rock detachment conditions in designing rockfall protection structures. Natural Hazards, 2016, 81 (1), pp.365-385. 10.1007/s11069-015-2084-0 . hal-01987688

\section{HAL Id: hal-01987688 \\ https://hal.science/hal-01987688}

Submitted on 16 Aug 2021

HAL is a multi-disciplinary open access archive for the deposit and dissemination of scientific research documents, whether they are published or not. The documents may come from teaching and research institutions in France or abroad, or from public or private research centers.
L'archive ouverte pluridisciplinaire HAL, est destinée au dépôt et à la diffusion de documents scientifiques de niveau recherche, publiés ou non, émanant des établissements d'enseignement et de recherche français ou étrangers, des laboratoires publics ou privés.

\section{다)(1) $(5$}

Distributed under a Creative Commons Attribution - NonCommerciall 4.0 International 


\title{
Accounting for the variability of rock detachment conditions in designing rockfall protection structures
}

\author{
Franck Bourrier $^{1,3} \cdot$ Julien Baroth $^{3,4} \cdot$ Stéphane Lambert $^{2,3}$
}

\begin{abstract}
This study is based on the analysis of the residual rockfall hazard at the elements at risk and accounts for the variability of the rock release parameters influencing the trajectory. The design of protection structures is conducted in two phases: a functional design phase consisting of quantifying the structure height from the rock passing height distribution and a structural design phase where the structure required capacity is assessed from the rock passing energy distribution. This framework is used on a well-documented study site for identifying the effects of the definition of the rocks release conditions, limited to the rock volume and falling height, on the design and efficiency of protection fences. The rock volume is modeled using a random variable, with different probabilistic laws. A probabilistic method is also used to analyze the effect of the rock volume distribution. These sensitivity analyses are conducted using a point estimate method for saving computation time. In this work, the initial falling height is shown to have a negligible influence on both the functional and structural designs of the fence. On the contrary, the rock volume range appears to be the leading parameter. The influence of the distribution law is shown to be of second order. The proposed approach may be extrapolated to other uncertain or variable parameters, as well as to other types of passive rockfall protective structures.
\end{abstract}

Keywords Rockfall protection structures · Trajectory simulations · Probabilistic design · Sensitivity analysis

Franck Bourrier

franck.bourrier@irstea.fr

Irstea, UR EMGR, 38402 St Martin d'Hères Cedex, France

Irstea, UR ETGR, 38402 St Martin d'Hères Cedex, France

3 Univ. Grenoble Alpes (UGA), 38402 Grenoble Cedex, France

4 3SR, CNRS UMR 5521, 38000 Grenoble Cedex, France 


\section{Introduction}

In the field of natural hazard management, the reliability of protective structures subjected to various kinds of phenomena, such as fire, flooding and earthquakes, relies on the capacity of these measures to reduce or suppress the hazard at the elements at risk (Guzzetti et al. 2004; Corominas et al. 2005; Agliardi et al. 2009; Volkwein et al. 2011; Mignelli et al. 2012). As for rockfall, the specific risk $R$ for the element at risk (housing, roads, etc.) to be damaged by a rock can be expressed as:

$$
R=\int_{e_{k}} P_{r}\left(e_{k}\right) V\left(e_{k}\right) \mathrm{d} e_{k}
$$

where $P_{r}\left(e_{k}\right)$ is the probability for the rock to reach the element at risk with an impact energy $e_{k}$ and $V\left(e_{k}\right)$ is the vulnerability of the element at risk depending on the impact energy $e_{k}$ of the rock. Although vulnerability functions exist for specific elements at risk (Agliardi et al. 2009; Mavrouli and Corominas 2010), integrating vulnerability remains difficult in practice mainly due to the lack of knowledge on the structures response to block impact (buildings, houses, etc.).

Alternatively, the rockfall hazard at the element at risk can be characterized in terms of probability for a block reaching the element at risk for any impact energy $e_{k}$, called global reach probability $P_{R}$ :

$$
P_{R}=\int_{e_{k}} P_{r}\left(e_{k}\right) \mathrm{d} e_{k}
$$

If information concerning vulnerability is missing, the global reach probability can be used for defining a site protection strategy, with the aim of reducing the hazard down a target residual value (Lambert et al. 2013). Such a strategy definition accounts for cost-benefit criterion (Eckert et al. 2008) and considers different countermeasures among which civil engineering protective structures.

This approach principally concerns passive rockfall countermeasures, like embankments or net fences, aiming at stopping rocks while descending the slope toward the at-risk elements. A protection strategy based on passive countermeasures requires finding the optimum in terms of structure location and structure design (Lambert and Bourrier 2013). This optimum can be evaluated based on the reach probability reduction derived from the structure construction (Lambert et al. 2013). Constraints due to accessibility, construction time and more generally budget are also accounted for. The structure design consists of two different phases: the first aiming at defining the required height for intercepting the blocks, while the second aiming at assessing the structure capacity in withstanding the impact and thus dissipating the block kinetic energy. Both these facets are based on rockfall propagation simulation results, namely the block passing height and its kinetic energy.

Rockfall propagation simulation tools play a major role in rockfall risk management. These tools provide run out distances, blocks kinetic energies and passing heights along the slope as required for land use planning and protective structure design. The rocks propagation is classically modeled as a stochastic process with respect to the rocks detachment conditions, the rocks size and shape, the slope topography and the slope surface materials (Bourrier et al. 2009a). The stochasticity associated with these different parameters in particular aims at accounting for the uncertainties related to their estimation. Most of these parameters are extremely variable with space, and some are difficult to assess. 
More specifically to the release area and block characteristics, the variable parameters are the block shape, its volume and its initial position, including altitude. In addition, information related to their fragmentability during rockfall may be given. In some cases, the values affected to these parameters and the associated variability are determined based on intensive investigations conducted on the cliffs (Ferrero et al. 2011) or based on previous events (Agliardi et al. 2009). In practice, the variability of all release area and block characteristics is rarely considered or integrated when conducting rockfall simulations. It is even less considered when designing protective structures. Most often, only the location of the release point, the size and the shape of the rocks are considered. These parameters are quantified from site observation by an expert. The shape of the block is generally determined using generic terms like "compact," "flat" or "angular." Except for the case where the risk is due to a single block, the volume is generally attributed a range. In case of a cliff, the maximal altitude is generally considered for the initial position, but a range may also be considered.

The literature dealing with the way to use rockfall trajectory simulation results for the design of protective structures is rather poor, in particular concerning the block passing height and kinetic energy. Sometimes, maximum values are considered, but most often quantiles in the 95-99\% range are proposed and recommended (Peila and Ronco 2009; UNI11211-4 2012; Christchurch City Council 2013; ONR 2013). For the design of the structure, these quantiles values are sometimes ponderated by safety coefficients depending on the frequency of the reference event, on the level of consequences in case of structure failure, and depending on the way some of the parameters used for the rockfall simulation have been determined (UNI11211-4 2012; ONR 2013). These coefficients were defined following the principles of the Eurocodes and should thus integrate the uncertainties associated with the parameters involved in the design. Also, these recommendations give the way to consider these parameters in relation to the worldwide used rockfall protection fences approval guideline ETAG 027 (EOTA 2013).

This paper addresses some key questions associated with the use of rockfall trajectory simulations for protective structures design purpose. It is based on a case study consisting in the design of a protection fence on a largely documented study site on which real-scale rockfall experiments were conducted (Dorren et al. 2006). The rockfall trajectories are modeled with Rockyfor3D (Bourrier et al. 2009a). Besides, the use of a point estimate method (PEM) is proposed for reducing computation times associated with the investigation of the influence of the variable parameters. Simulations are conducted with the aim of investigating the influence of two release condition parameters: the initial falling height and the block volume. The results are discussed placing an emphasis on the influence of these two parameters and on the use of statistical indicators of their distributions, i.e., quantiles, for the practical design of protection structures.

\section{Materials and methods}

\subsection{Case study}

The study site chosen for illustrating the proposed approach corresponds to a full-scale rockfall experimental site located in an avalanche track in the Forêt Communale de Vaujany in France $\left(45^{\circ} 12^{\prime} \mathrm{N}, 6^{\circ} 3^{\prime} \mathrm{E}\right)$. The site covers an alpine slope denuded of trees ranging from 1200 to $1400 \mathrm{~m}$ above sea level. It is $100 \mathrm{~m}$ wide and $570 \mathrm{~m}$ long (distance 

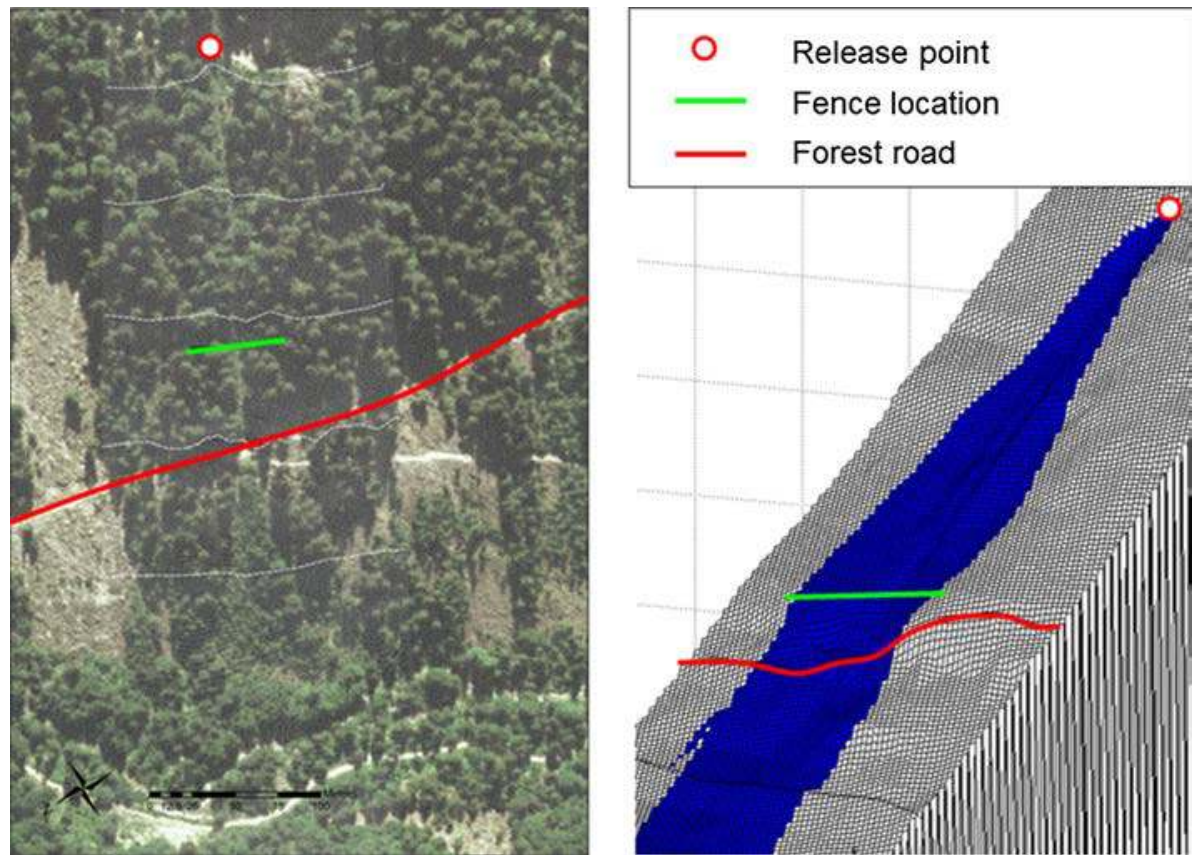

Fig. 1 Description of the case study (Vaujany, France, $45^{\circ} 12^{\prime} \mathrm{N}, 6^{\circ} 3^{\prime} \mathrm{E}$ ). Photograph (left), numerical model (right). Relative positions of release point, fence location, forest road

between the starting point and the lower forest road, measured along the slope) with a mean gradient of $38^{\circ}$ (Fig. 1).

The element at risk considered in this study is the forest road located at mid-slope of the study site (Fig. 1). To protect this element at risk, the choice is made to install a rockfall protection fence uphill the element at risk. The fence was located $235 \mathrm{~m}$ from the release point of the rocks. This specific location was chosen because rock velocities, kinetic energies and passing heights were measured there during previous rockfall experiments held in the site (Dorren et al. 2006). These measurements allowed calibrating Rockyfor3D, the rockfall simulation code used for this study.

\subsection{Rockfall simulation code Rockyfor3D}

The rock propagation simulations were conducted using the code Rockyfor3D. This wellknown and widespread tool is used to model the propagation of rocks along mountain slopes and, in particular, the passing heights and kinetic energies of the rocks at the potential location of the protection structures. Rockyfor3D simulates the 3D propagation of the rocks as a succession of free flights through the air and rebounds on the soil. The rock propagation is modeled through a rasterized digital terrain model which can be seen as a matrix giving the mean elevation of each square cell representing the terrain. The rebound calculation is conducted in two independent phases. First, the deviation of the block trajectory after the rebound is assessed, and second, the block reflected velocity is calculated. This modeling approach was shown to provide satisfying results at the slope scale (Dorren et al. 2006; Bourrier et al. 2009a). 
A series of rockfall simulations consists of releasing from each user-defined departure cell a given number of rocks one after the other. Output results from the simulations are used to build the complete statistical distribution of the rock trajectories when entering a user-defined set of cells, corresponding to the fence location in this case. The setup of a rockfall simulation series requires defining a set of properties related to the slope surface, the rocks and the release conditions (rock volume, departure location and initial falling height, in particular). Finally, RockyFor3D allows for simulating the trajectory of blocks with a volume sampled from an uniform distribution law over a user-defined volume range.

For this study, the code parameters describing the slope surface and the topography are set at the same values as in the calibration phase of the code (Dorren et al. 2006). Only the values of the parameters related to the rock release conditions, namely rock volume and initial falling height, are changed.

\subsection{Sensitivity analysis using a point estimate method}

The effects of the rock release conditions on the design of the fence are analyzed for the case study exposed in Sect. 2.1. The analysis focuses on the effect of changing the distribution of the rock volume on the design of the fence.

For that purpose, we are interested in the distributions of the rock passing height, denoted $z_{1}$, and of the kinetic energy, denoted $z_{2}$, at the fence location. Using cumulative distribution functions of these quantities allows deducing probabilities of exceeding limits heights and energies. Such an analysis can be done by performing a large set of rockfall simulations for values of the rock volume (denoted $y$ ) sampled in its distribution.

With the aim of saving computation time comparing various distributions of $y$, we propose herein a strategy to limit the total number $N$ of simulations for calculating the required probabilities.

First, we consider histograms divided in $r_{1}$ and $r_{2}$ classes, in $\Omega_{1}=\left[z_{1, \operatorname{Min}}, z_{1, \mathrm{Max}}\right]$ and $\Omega_{2}=\left[z_{2, \text { Min }}, z_{2, \text { Max }}\right]$ with relative frequencies $\left(f_{1, i}\right)_{i=1, \ldots, r_{1}},\left(f_{2, i}\right)_{i=1, \ldots, r_{2}}$. For the sake of simplicity, we will consider in the following $r=r_{1}=r_{2}$ and generic frequencies $\left(f_{i}\right)_{i=1, \ldots, r}$.

The number of simulations is reduced by approximating frequencies $f_{i}$ using a quadrature rule, such that

$$
f_{i} \approx \hat{f}^{i}=\sum_{j=1}^{k} f_{i, j}\left(y_{j}\right) \omega_{j}
$$

The frequencies $f_{i, j}$ are obtained from $k$ sets of $N_{j}$ rockfall simulations using selected values of rock volumes $\left(y_{j}\right)_{1 \leq j \leq k}$. The specific weights $\omega_{j}$ depend on the rock volume distribution and on $k$. Values of weight can be found, for example, in (Abramowitz and Stegun 1970) and are detailed in "Appendix 1," for $j=1, \ldots, k$, with $k=3,4,5$, for 2 distributions (uniform and Gaussian).

These quadrature rules are more generally used to approximate statistical moments and integrals ("Appendix 1"). Such a method can be considered as one of the so-called PEM, already used in various fields, e.g., geotechnics (Schweiger 2001) or nuclear engineering (Millard et al. 2000).

In the following application, we will show that using $k=5$ sets of $N_{j}$ rockfall simulations, with $N_{j}=N / 5$, allows estimating the effect of different distributions of $y$ (uniform, Gaussian, beta) on the fence design. 


\subsection{Principles for the structure design based on rockfall simulations}

In the frame of this study, it is proposed to consider the reach probability at the element at risk as the main criterion for the design of the fence. The hazard downhill the protective structure once constructed is called residual hazard.

The element at risk is protected by a fence with an height $h_{s}$ uphill in the slope (Fig. 2). The fence is long enough to intercept all the blocks, whatever their lateral dispersion. The fence capacity in terms of block kinetic energy at failure is noted $e_{k}^{*}$.

For given rock size and shape, the release of a rock is associated with a probability $P_{D}$ that can be deduced from research studies on specific sites (Hantz 2012). Alternatively, $P_{D}$ can be assumed equal to 1 if the analysis aims at designing a structure given that the rock release occurs, in particular. The latter approach was chosen in this study.

As illustrated in Fig. 3, the rockfall propagation process while in the presence of a protective fence can lead to different outcomes, called events in the following:

- $\boldsymbol{E}_{\boldsymbol{H I}}$ : rock coming to a halt on the slope, before reaching the fence;

- $\boldsymbol{E}_{\boldsymbol{H} \boldsymbol{I}}^{-}$: rock reaching the fence;

- $\boldsymbol{E}_{\boldsymbol{L}}$ : rock reaching the fence with a passing height $z>h_{s}$ leading to the overtopping or lob of the fence;

- $\boldsymbol{E}_{\boldsymbol{F}}$ : rock reaching the fence with a passing height $z \leq h_{s}$ and with an impact energy $e_{k}>e_{k}^{*}$ : leading to the puncture of the fence (or failure);

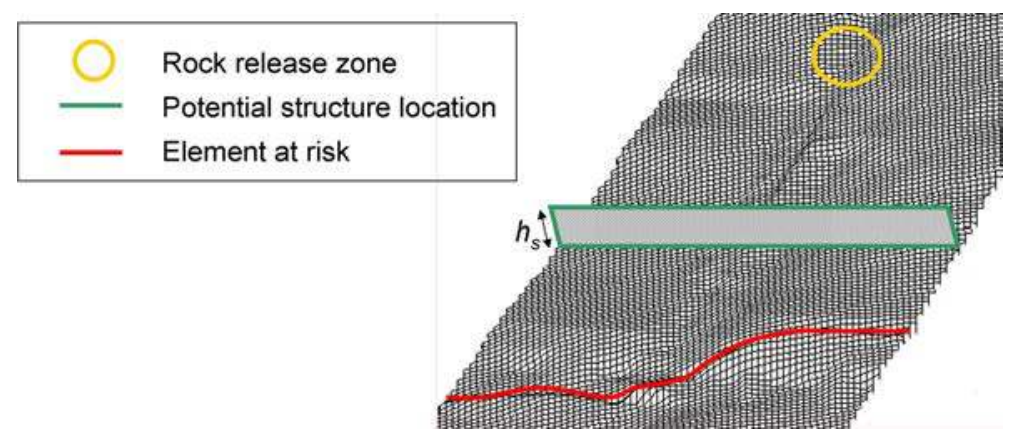

Fig. 2 Typical case study in the context of protection of linear stakes against rockfall

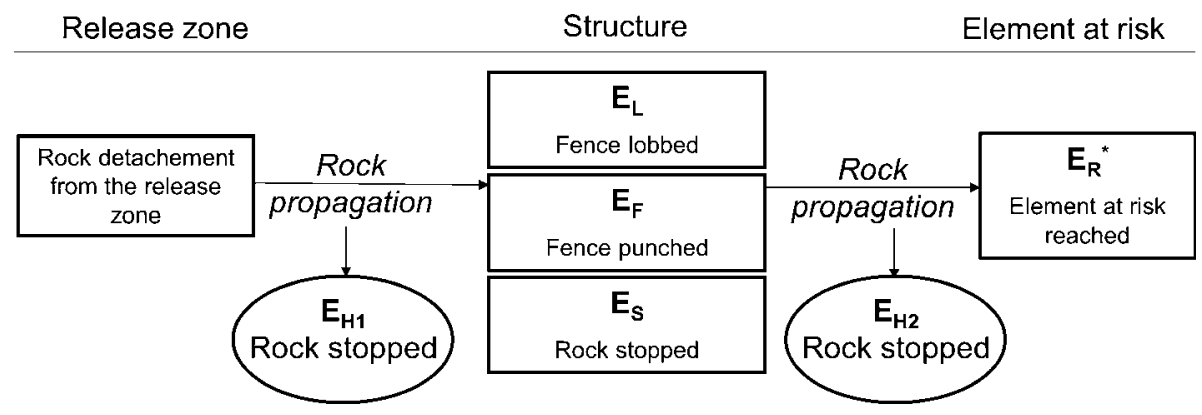

Fig. 3 Chain of events potentially occurring during a rockfall in a study site where the element at risks are protected by rockfall fences 
- $\boldsymbol{E}_{S}$ : rock stopped by the fence, thus having a passing height $z \leq h_{s}$ and an impact energy $e_{k}<e_{k}^{*}$

- $\boldsymbol{E}_{\boldsymbol{H} 2}$ : rock coming to a halt on the slope, downhill the protection structure but before reaching the element at risk;

- $\boldsymbol{E}_{\boldsymbol{R}}^{*}$ : rock reaching the element at risk.

The probability $P_{\mathrm{L}}$ that a rock overpasses the fence is calculated from the distribution of the rocks passing height $z$ at the fence location. The aim being to focus on the fence design, this probability is calculated with reference to the number of blocks reaching the cells associated with the fence location. Thus, it is given by $P_{\mathrm{L}}=P\left(\boldsymbol{E}_{\boldsymbol{L}} / \boldsymbol{E}_{\boldsymbol{H} \boldsymbol{1}}^{-}\right)$. Similarly, the probability $P_{\mathrm{F}}$ of the fence failure should be determined considering the blocks reaching the fence with a passing height less than the fence height and an impact energy $e_{k}>e_{k}^{*}$ : $P_{\mathrm{F}}=P\left(\boldsymbol{E}_{\boldsymbol{F}} / \boldsymbol{E}_{\boldsymbol{H} 1}^{-}\right)$. Determining the probability $P_{\mathrm{F}}$ thus requires knowing both the passing height $z$ and passing energy $e_{k}$ of all rocks reaching the fence location.

Finally, among the rocks that overpass or lead to the failure of the fence not all rocks reach the element at risk because they can be stopped before. Consequently, the probability $P_{\mathrm{L}}+P_{\mathrm{F}}$ and the probability $P_{R}=P\left(\boldsymbol{E}_{\boldsymbol{R}}^{*}\right)$ for the rock to reach the element at risk are different. These two probabilities can be assumed equal for the sake of simplicity, leading to neglect $P\left(\boldsymbol{E}_{\boldsymbol{H} 2}\right)$. This assumption is conservative and it is true when the protection structure is located near the element at risk. In the following, this assumption will be used provided that it does not change the principles of the approach proposed and the tendencies in the results obtained.

The design of a rockfall protection fence at a given location is therefore based on the estimation of the probabilities $P_{\mathrm{L}}$ and $P_{\mathrm{F}}$. We propose to call functional design of the fence, the choice of a fence height based on an acceptable probability of lob $P_{\mathrm{L}}$, and structural design of the fence, the choice of a fence capacity based on an acceptable probability of failure $P_{\mathrm{F}}$. Both are presented in the following.

The proposed approach is based on rockfall trajectory simulation results in the absence of structure. It relies on the assumption that the blocks trajectories before reaching the structure are not modified by the structure construction. Under this assumption, the integration of the protection structure does not significantly changes the topography and the material properties of the slope, which is the case for net fences but not for embankments (Lambert and Bourrier 2013).

Besides, the approach is developed considering a predefined structure location. In reality, the fence location choice may depend on the functional and structural designs results, in addition to possible site-specific constraints.

\subsubsection{Functional design}

The aim of the functional design is to determine the fence interception height, or fence height, once its location is defined. In a functional approach, any rock passing below this height is considered stopped. The fence height is considered with respect to the vertical. It is based on the blocks gravity center passing height as obtained from the trajectory simulations. For design purpose, one may add to this value the block radius or a freeboard or consider a safety coefficient (Peila and Ronco 2009; UNI11211-4 2012; ONR 2013).

The functional design consists in defining the fence height so that the lob probability $P_{\mathrm{L}}$ is lowered down to a target value called functional residual hazard (FRH) in the following. This means that, for example, the height of a fence with a $5 \%$ FRH equals the passing height of the gravity center of $95 \%$ of the blocks reaching the fence location. 


\subsubsection{Structural design}

The structural design of the fence is conducted once the height of the fence has been determined, during the functional design phase. During the structural design phase, only the rocks impacting the fence are considered. The aim of this phase is to design the fence under the variable loading as provided by the trajectory simulations. The fence properties are determined so that the probability $P_{\mathrm{F}}$ that a rock induces fence failure is lowered to a target residual value called structural residual hazard (SRH).

For the structural design, either numerical model of the structure or reglementary rules, as for instance the ETAG 027 (EOTA 2013), can be used to assess the maximum impact energy the fence is able to withstand. Determining the probability for the fence failure under variable loading conditions using complex numerical models requires performing large amount of simulations to provide statistically relevant results (Bourrier et al. 2015). This point is a crucial limitation to the use of numerical models of the impact on the fence for that purpose. Besides, the mechanical response of a net fence is highly dependent on its type.

As the focus of this study is on the general principles of structure design, the choice was made not to consider a specific one. The results will be presented and discussed in terms of fence capacity. The required fence capacity is taken equal to the maximum block kinetic energy that the fence can withstand. Contrary to what is sometimes recommended, no safety coefficient is considered (UNI11211-4 2012; ONR 2013).

\subsubsection{Calculation of the residual hazards from rockfall simulations}

The methodology for the design of a protection structure exposed above is based on the design of the structure height (resp. capacity) corresponding to a target functional (resp. structural) residual hazard.

The functional design relies on the assessment of the functional residual hazard depending on the fence height. The functional residual hazard corresponds to the probability for the rock to pass over a given height. This probability is characterized from rockfall simulations using the cumulative distribution function of the passing height at the fence location.

Similarly, the structural residual hazard depends on the fence capacity, i.e., maximum impact energy it is able to withstand. It is the probability for rocks to pass at fence location with an energy larger than the fence capacity. The cumulative distribution function of the kinetic energy at the fence location is used to characterize this probability.

\subsection{Simulation series and parameters}

All released rocks are assumed to be spherical in shape, and the rock density is set at $2600 \mathrm{~kg} / \mathrm{m}^{3}$. The rock release point is located as for the previous experiments in the site (Dorren et al. 2006).

Reference release conditions are first defined corresponding to a rock volume set at $1.25 \mathrm{~m}^{3}$ and an initial falling height set at $5 \mathrm{~m}$. The sensitivity of the results to the number of released rocks is addressed. Second, the influence of the variability of the release conditions on the structural and functional design is analyzed. For that purpose, the rock volume is assumed to vary on a domain $\Omega=\left[0.5 \mathrm{~m}^{3} ; 2 \mathrm{~m}^{3}\right]$. First, a uniform distribution of the rock volume was assumed, and second, different distributions of this quantity were 
Table 1 Rock volume distributions tested (uniform, Gaussian, beta)

\begin{tabular}{lll}
\hline Variable & Law & Parameters $\left(\mathrm{m}^{3}\right)$ \\
\hline$Y_{1}$ & Uniform $U\left(y_{\min }, y_{\max }\right)$ & $y_{\min }=0.5 ; y_{\max }=2$ \\
$Y_{2}$ & Gaussian $N\left(\mu_{Y_{3}}, \sigma_{Y_{3}}^{2}\right)$ & $\mu_{Y_{3}}=1.25 ; \sigma_{Y_{3}}=0.59$ \\
$Y_{3}$ & Beta $(\alpha, \beta)$ & $\mu_{Y_{5}}=1.25 ; \sigma_{Y_{5}}=0.43$ if $(\alpha=\beta=1)$ \\
\hline
\end{tabular}

tested (Gaussian and beta distributions-cf. Table 1). In addition, simulations are performed for rocks initial falling heights ranging from 1 to $50 \mathrm{~m}$. It is worth highlighting that these two parameters are independents, allowing for investigating their influence separately.

\section{Results}

\subsection{Design for deterministic release conditions}

This section presents the results of the simulation for the reference case, i.e., a $1.25 \mathrm{~m}^{3}$ block with an initial falling height of $5 \mathrm{~m}$. Results based on simulations increasing the number of released rocks are provided. One can note that for any simulations presented afterward, more than $90 \%$ of the released blocks reached the fence location [i.e., $\left.P\left(\boldsymbol{E}_{\boldsymbol{H} \boldsymbol{I}}\right)=0.1\right]$. Then, the influence of both the rock volume and rock initial falling height is addressed independently and considering these parameters deterministic.

Whatever the number of released rocks, decreasing the functional hazard induces an increase in the required fence height (Fig. 4a). Significant differences with the 100,000 release curve are observed for small number of rock releases and for small FRH values. For example, the required fence height corresponding to a $1 \%$ FRH is $2.71,2.42$ and $3.08 \mathrm{~m}$ when deduced from simulations of 100,000-1000 and 100 blocks releases, respectively. The difference between the extreme values is as high as $28 \%$. Smaller differences are observed increasing the target FRH: the difference in fence height between the extreme

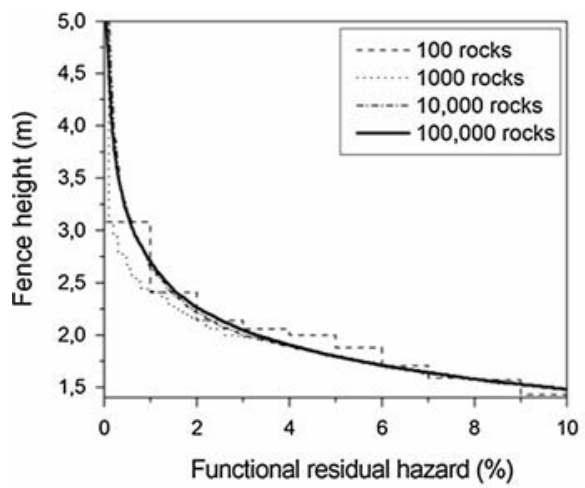

(a)

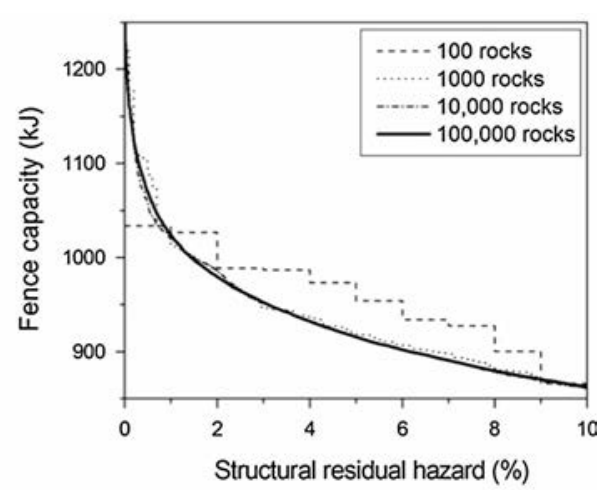

(b)

Fig. 4 Influence of the number of rock releases on the functional (a) and structural (b) residual hazard depending on the fence height and impact energy, respectively 
Fig. 5 Cumulative distribution function of the structural residual hazard at the fence location for different fence heights $h_{s}$

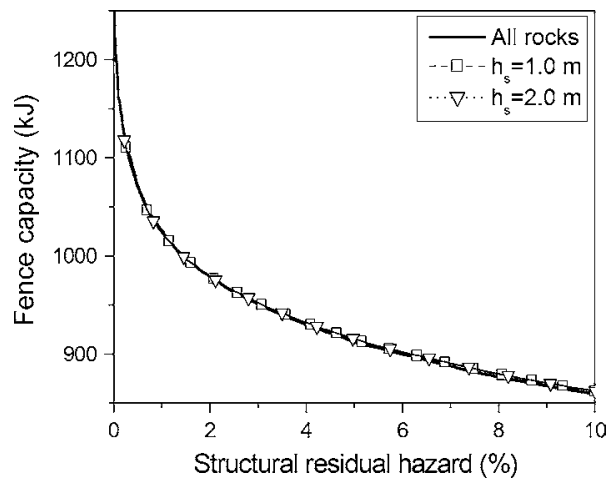

cases vanishes to less than $5 \%$ for a FRH of $4 \%$. Despite the rather fast convergence observed, the choice is made to use 100,000 rock releases per rockfall simulation set to insure that the relationship between the FRH and the required fence height has reached stable values.

The structural design phase consists in analyzing the distribution of the kinetic energy of the rock impacting the fence. This would require excluding the analysis all the blocks passing above the fence, that is having a passing height higher than the fence height. Also, questions concerning the way to consider the blocks which center of gravity is just above the fence but touching the fence may rise. However, in the example treated, the fence capacity vs. SRH curves exhibit very small differences if only the rocks passing under a predefined height $h_{s}$ are considered compared to the case where all the blocks are considered (Fig. 5). In the following, we will thus use the distribution of the kinetic energy of all rocks passing at the fence location for the structural design instead. It does not induce significant changes in this case study, while it reduces complexity in the processing of the rockfall simulations.

The results obtained for the required fence capacity depending on the structural residual hazard (Fig. 4b) highlight significant similarities in terms of convergence with those obtained for the evolution of the fence height depending on the FRH (Fig. 4a). When increasing the number of blocks releases, the required fence capacity rapidly converges to the value obtained with 100,000 simulations. A total of 1000 releases are sufficient to obtain a quasi identical curve. Besides, the difference in fence capacity between the two extreme cases is typically $5 \%$ over the $2-8 \%$ SRH range. Despite this fast convergence and as for the distribution of the rocks passing height, the choice is made to use 100,000 rock releases per rockfall simulation to insure that all quantiles of the kinetic energy distribution have reached stable values.

Besides, both curves presented in Fig. 4 reveal a significant change in design parameter over the $0-5 \%$ residual hazard range. The fence capacity for a $1 \%$ SRH is 1.12 times that obtained for the $5 \%$ SRH. More notably, the fence height for a $1 \%$ FRH is 1.6 times that obtained for the $5 \%$ FRH. This suggests that the design based on the $95 \%$ quantile of both the passing height and energy of the blocks may be unconservative.

The influence of the values of the release conditions parameters considered deterministic is explored independently. A total of 100,000 rockfall simulations are performed for different initial falling heights ranging from 1 to $50 \mathrm{~m}$ and then for different rock volumes ranging from 0.5 to $2 \mathrm{~m}^{3}$ all other simulation parameters being unchanged. The initial 


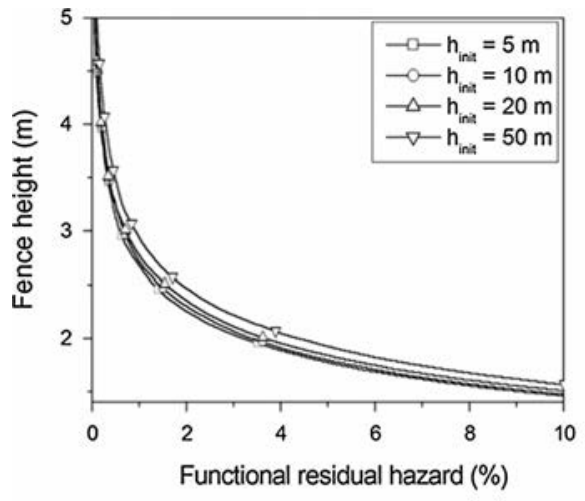

(a)

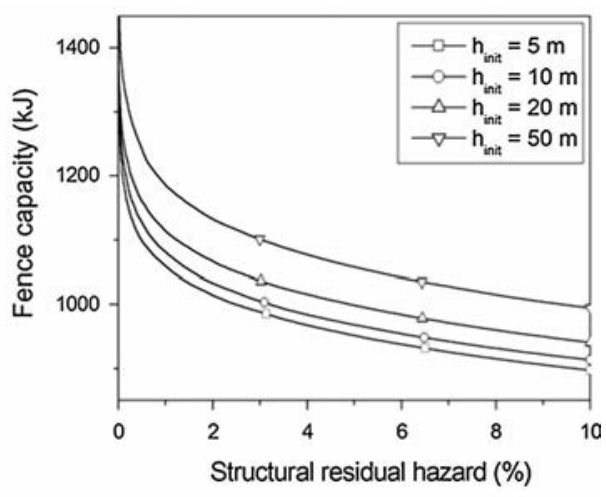

(b)

Fig. 6 Evolution of the required fence height and capacity for different rock initial falling heights $h_{\text {init }}$ as a function of the FRH and SRH (a, b respectively)

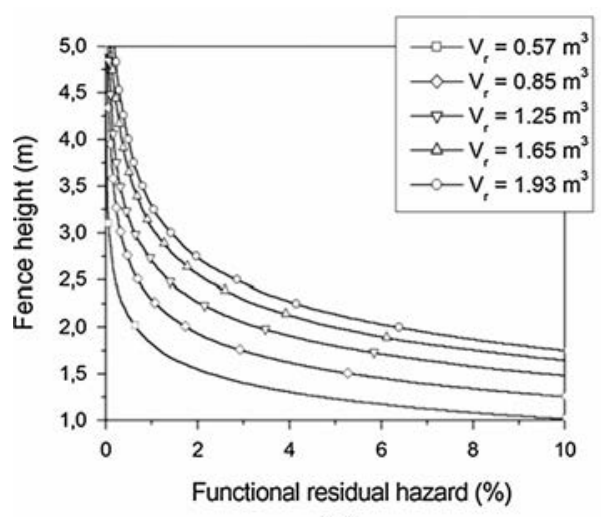

(a)

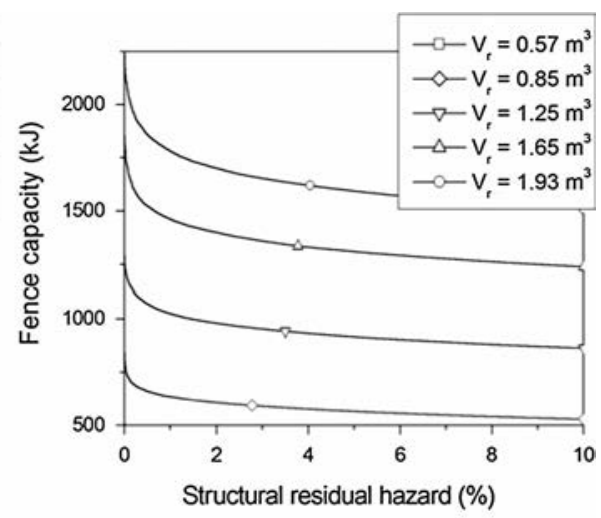

(b)

Fig. 7 Evolution of the required fence height and capacity for different rock volumes $V_{\mathrm{r}}$ as a function of the FRH and SRH (a, b respectively)

falling height of the rock has almost no influence on the required fence height, for any FRH value (Fig. 6). A clear difference is observed on the fence capacity, with a $17 \%$ difference between the extreme cases considering a $2 \%$ SRH value for example. On the contrary, the sensitivity to the rock volume is much higher for both the fence height and capacity (Fig. 7). For a $2 \%$ FRH, a ratio of almost 2 is observed for the required fence height between the two extreme cases. For a $2 \%$ SRH, a ratio of almost 3 is observed for the required fence capacity between the two extreme cases. Finally, all the graphs plotted in Figs. 6 and 7 show a significant variation of design parameters over the 0-5\% hazard range, with ratios in the same order of magnitude as that observed in Fig. 4.

\subsection{Accounting for the variability of the release conditions}

Considering the results presented in the previous section, the choice was made to limit the number of variable release condition parameters to the only rock volume. The analysis of 
the influence of the statistical distribution of this parameter on the fence design is conducted considering a deterministic rock release height set at $5 \mathrm{~m}$ and a rock volume ranging from 0.5 to $2 \mathrm{~m}^{3}$. First, a uniform distribution is considered before investigating the interest in considering different statistical distributions while using results based on a PEM.

\subsubsection{Design for uniformly distributed rock volumes}

These simulations were conducted considering 100,000 blocks with a volume randomly sampled in a uniform distribution as proposed by RockyFor3D. The results from these direct simulations allow estimating the influence of considering the rock volume as a random variable on the functional and structural residual hazards as compared to results obtained for a single rock volume value set at the mean value of the volume range (i.e., $1.25 \mathrm{~m}^{3}$ ) (Fig. 8).

The statistically based design of the fence height is not changed when considering a variable rock volume (Fig. 8a). On the contrary, the fence capacity is very different depending whether the rock volume variability is accounted for or not (Fig. 8b). For example, the $5 \%$ (resp. $1 \%$ ) structural residual hazard correspond to a required fence capacity of $1300 \mathrm{~kJ}$ (resp. $1500 \mathrm{~kJ}$ ) compared to $900 \mathrm{~kJ}$ (resp. $1000 \mathrm{~kJ}$ ) if the rock volume is not considered as a random variable. Considering the medium volume is not conservative, leading, for example, to a required fence capacity $40 \%$ lower than obtained for the uniform distribution, in the $5 \%$ SRH case. Specific analyses of the influence of the rock volume distribution on the structural design of the fence are thus crucial to avoid installing fences not having the capacity to withstand the impacts.

\subsubsection{Comparison with a point estimate method results}

The analysis of the effect of the rock volume distribution or variation range on the residual hazards potentially requires doing many simulations. This has motivated using a PEMbased approach exposed in Sect. 2.3.

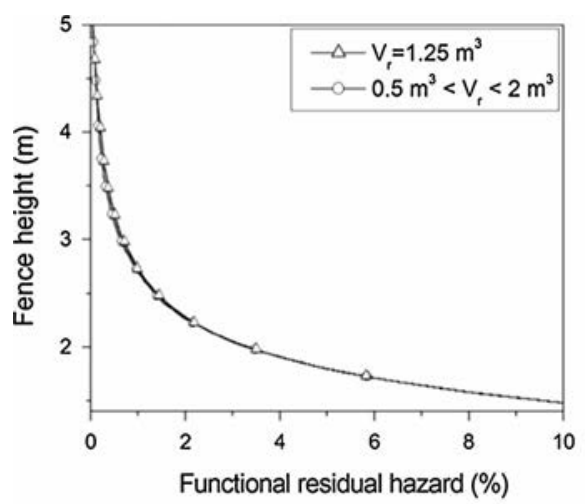

(a)

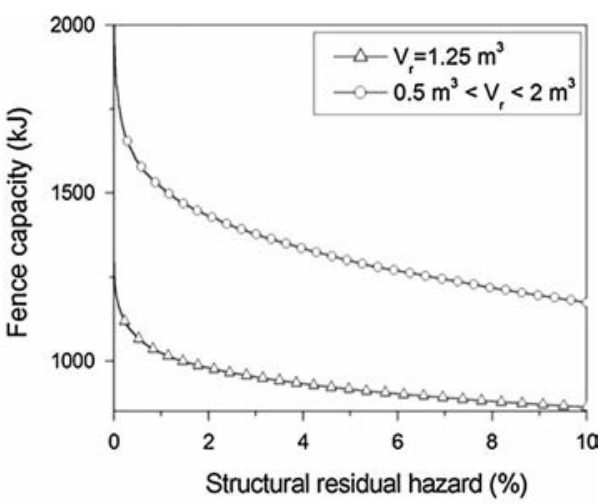

(b)

Fig. 8 Comparison between the fence height versus FRH curve and fence capacity versus SRH curve (resp. a, b), for a uniform distribution of the rock volume ranging from $0.5 \mathrm{~m}^{3}$ to $2 \mathrm{~m}^{3}$ and for a rock volume set at $1.25 \mathrm{~m}^{3}$ 
Table 2 Values of rock volumes $\left(\mathrm{m}^{3}\right)$ for each set of rockfall simulations

\begin{tabular}{llllll}
\hline Points & $y_{1}$ & $y_{2}$ & $y_{3}$ & $y_{4}$ & $y_{5}$ \\
\hline Volumes $\left(\mathrm{m}^{3}\right)$ & 0.57 & 0.85 & 1.25 & 1.65 & 1.93 \\
\hline
\end{tabular}

Using this method, the functional and structural residual hazards are reconstructed from 5 simulation sets associated with $k=5$ constant values $y_{j}$ of the rock volume between 0.5 and $2 \mathrm{~m}^{3}$ (Table 2). Then, various distributions can be tested (Table 1), using the procedure detailed in "Appendix 2."

The method requires calibrating the number of classes required for a correct assessment of the fence height and fence capacity distributions. This calibration was conducted by comparison with the results from the direct simulations with uniformly distributed volumes as presented in the previous section. The simulations involved $N_{j}=20,000$ blocks releases per simulation set (i.e., per block volume $y_{j}$ as given Table 2), for a total of 100,000. The results show that in the context of this study, a minimum of $r=20$ classes have to be considered to provide a relevant calculation of the residual hazards (Fig. 9). A total of 50 classes will be considered in the following to ensure results relevance.

Then, the sensitivity of the PEM results on the number of blocks released on total was investigated and compared with the results of the direct simulations conducted using RockyFor3D (Fig. 10). The PEM appears relevant providing the total number of block releases is higher than 1000 rocks. In such a case, the difference in terms of fence height (resp. capacity) between the PEM results and the direct simulation results is less than 10 $\mathrm{cm}$ (resp. $40 \mathrm{~kJ}$ ) whatever the residual hazard considered. In a practical context, this difference is negligible.

Finally, the benefit in using a PEM in terms of computation time appears not significant. In fact, the advantage of a PEM is that, based on the same 5 simulation sets, different statistical distribution laws can be considered without conducting new rockfall simulation, just by changing the weights.

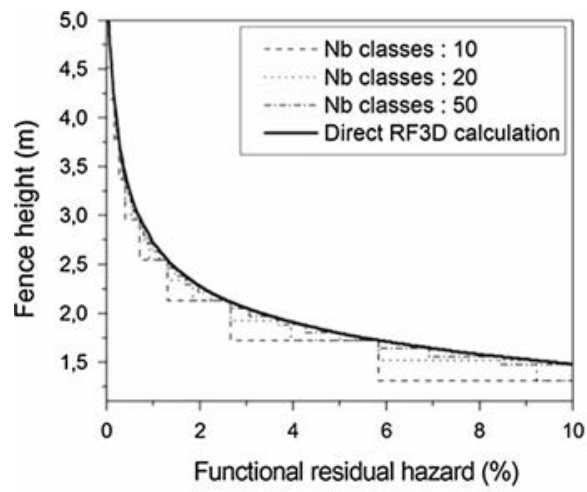

(a)

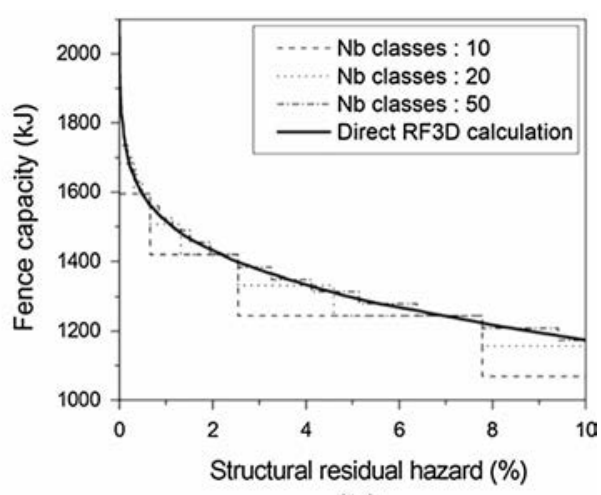

(b)

Fig. 9 Comparison between the evolutions of the functional (a) and structural (b) residual hazard for a uniform distribution of the rock volume ranging from 0.5 to $2 \mathrm{~m}^{3}$ obtained using direct analysis of rockfall simulations and the point method estimate method with different number of classes $N$ for $f_{i, j}$ 


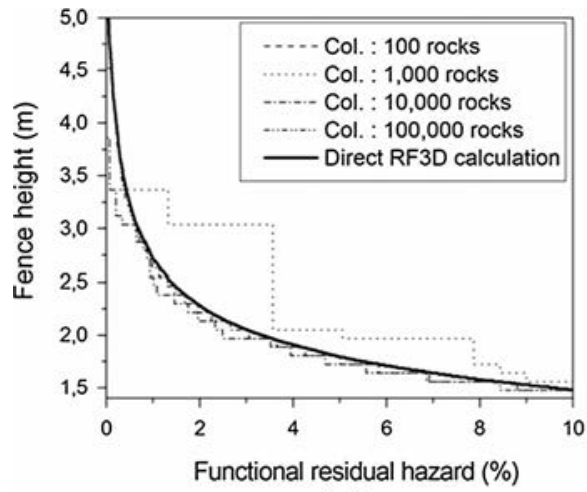

(a)

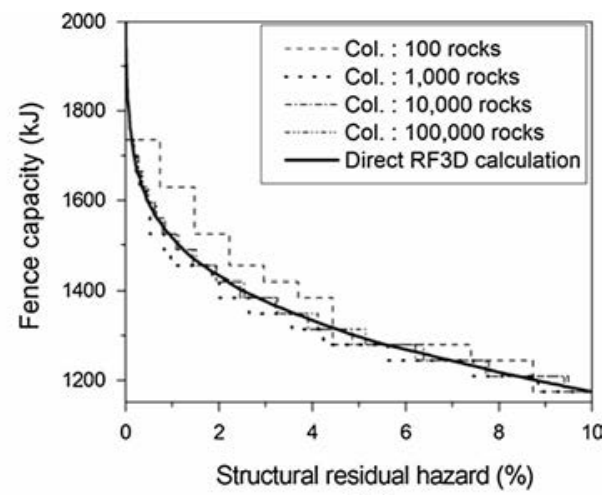

(b)

Fig. 10 Comparison between the evolutions of the functional (a) and structural (a) residual hazard for a uniform distribution of the rock volume ranging from $0.5 \mathrm{~m}^{3}$ to $2 \mathrm{~m}^{3}$ obtained using direct analysis of rockfall simulations and the point estimate method varying the number of blocks releases

\subsubsection{Analysis of the influence of the rock volume statistical distribution using a point estimate method}

Accounting for the variability of the rock volume using a uniform distribution law is a simple but certainly unrealistic approach. Different distributions may be considered to better fit with real sites. This can be conveniently conducted thanks to a PEM, by using the same set of rockfall simulations for all distributions. In this study, a Gaussian distribution and a beta distribution were considered (see Table 1). Different weights (see "Appendix 1") were used to calculate the functional and structural residual hazards from the same rockfall simulations as the one used for building the residual hazards curves for the uniform distribution (Fig. 11).

The results show that the fence height is not modified changing the rock volume distribution, contrary to the fence capacity (Fig. 12). For instance, a $5 \%$ (resp. $1 \%$ ) SRH requires a fence with a capacity ranging from 1100 to $1300 \mathrm{~kJ}$ (resp. 1300-1700 kJ) depending on the rock volume distribution considered, corresponding to a difference up to $30 \%$. These results show that not only the variation range but also the distribution of the rock volume can influence the fence capacity design. Finally, the uniform distribution appears rather conservative in terms of fence capacity.

\section{Discussion}

The initial falling height has been shown to have almost no influence on the design of the fence for the study site considered. This is primarily attributed to the energy dissipation during the first impacts on soil. Prior to the first impact, the block trajectory is vertical and the block incident angle with respect to the soil surface is high. This has been shown to favor energy dissipation compared to shallow impacts (Bourrier et al. 2009a, b). Thus, the first impact induces substantial decrease in the rock energy except for initial falling heights smaller than $5 \mathrm{~m}$, as shown in Table 3. On the contrary, for initial falling heights larger than $5 \mathrm{~m}$, the mean rock energy after the first $25 \mathrm{~m}$ of rock propagation is substantially 


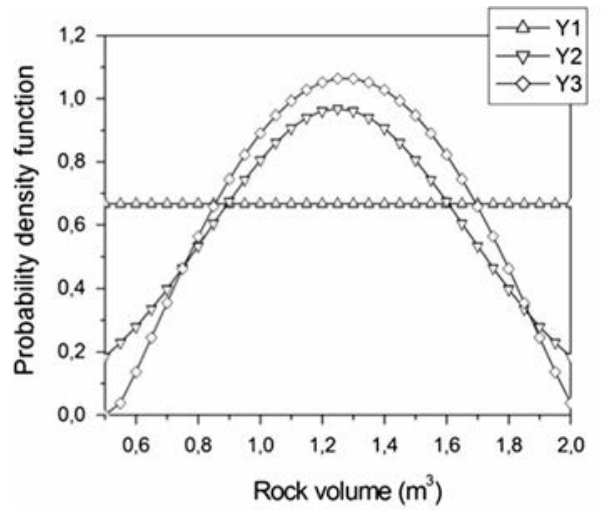

(a)

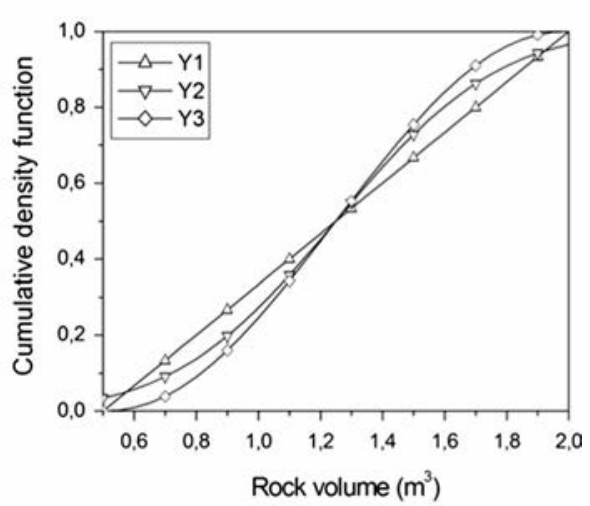

(b)

Fig. 11 Probability (a) and cumulative (b) density functions of the three rock volume distributions considered

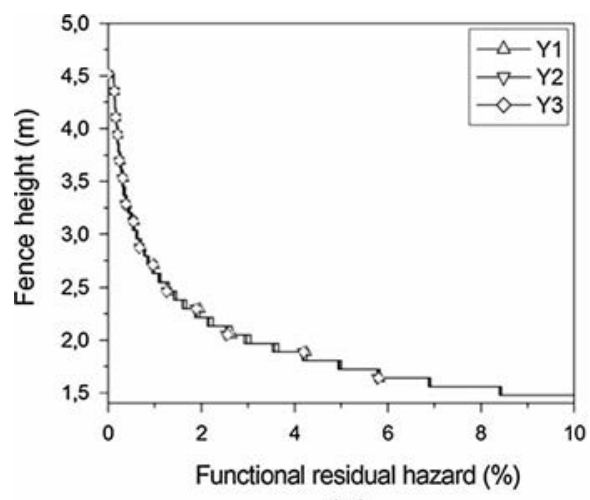

(a)

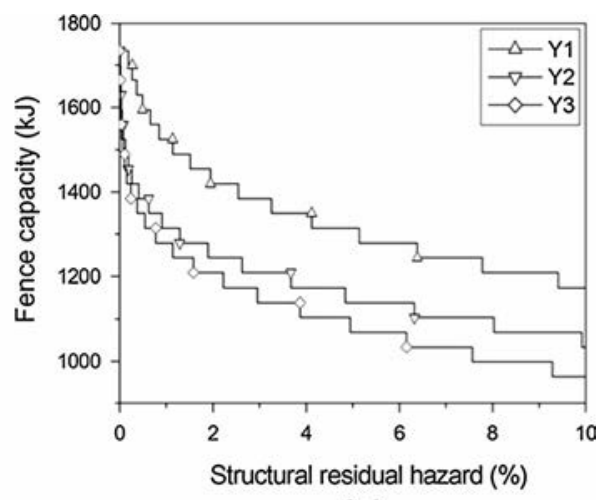

(b)

Fig. 12 Functional (a) and structural (b) residual hazards obtained for 3 distributions of the rock volume compared using the point estimate method

lower than the initial rock potential energy, i.e., than the rock kinetic energy just before the first impact. The differences between the mean rock kinetic energy for different initial falling heights decrease during the rock propagation to reach a value of $83 \mathrm{~kJ}$ after $225 \mathrm{~m}$ of rock propagation (Table 3 ). The practical consequence is that for vertical cliffs presenting a diffuse detachment risk, the uncertainties related to the determination of the rock initial falling height by expert knowledge are of minor influence if the fence to be designed is located far enough from the rock release area. However, this conclusion may be strongly depending on the topographical specificities of the site considered. Additional series of rockfall simulations should be carried out on different slope geometries to check whether this conclusion can be generalized. Such a detailed analysis can constitute a promising perspective for further researches.

On the contrary, the influence of the block volume on the fence design is much more important, and more particularly concerning its capacity. Except when the hazard results 
Table 3 Mean rock kinetic energy $(\mathrm{kJ})$ at different distances from the release point and for the different initial falling heights $h_{\text {init }}$

\begin{tabular}{lcrrrrr}
\hline \multicolumn{7}{l}{ Rock propagation distance $(\mathrm{m})$} \\
\cline { 2 - 6 } & 0 (before 1st impact) & 25 & 75 & 125 & 175 & 225 \\
\hline$h_{\text {init }}(\mathrm{m})$ & & & & & & \\
1 & 40 & 95 & 248 & 455 & 519 & 448 \\
2 & 74 & 100 & 253 & 474 & 555 & 496 \\
5 & 175 & 125 & 269 & 472 & 545 & 465 \\
10 & 342 & 146 & 299 & 512 & 596 & 495 \\
20 & 671 & 185 & 348 & 516 & 588 & 509 \\
50 & 1157 & 256 & 430 & 597 & 622 & 531 \\
Max. energy diff. (kJ) & 1117 & 161 & 182 & 142 & 103 & 83 \\
\hline
\end{tabular}

The simulation parameters are those used in Sect. 3.1

from a single and well-identified block, the volume is generally difficult to estimate in practice, with ranges in ratio up to $1-10$ on a same geological unit. With this respect, the volume range considered in this study may be considered small. However, considering either a deterministic or a uniformly distributed volume leads to a ratio of almost 2 (resp. 3) in terms of fence height (resp. capacity) based on a $2 \%$ residual hazard criterion. The rock volume evaluation is thus crucial to the structure design, and it is highly relevant to consider a volume range instead of a single volume. Currently available rockfall simulation tools often propose considering a uniformly distributed rock volume. Such a distribution has been shown to be conservative in terms of fence capacity, the fence height being much less sensitive to the volume distribution. Besides, the minor differences between the fence capacity curves depending on the volume distribution statistical laws suggest that the gross benefit derived from the improvement in these laws would be minimum. Indeed, the costs induced for obtaining precise enough volume distribution laws on specific sites would certainly not be compensated by the gains derived from the optimization of the fence.

A PEM-based approach has been considered for saving time in investigating the influence of the different parameters. More precisely, the PEM approach was used for investigating the influence of the rock volume distribution law. Comparison with direct simulations using RockyFor3D confirmed that, in case of a uniform distribution, the same results were obtained. Nevertheless, no real benefit in terms of computation time was derived. Indeed, using such approaches can allow decreasing computational costs by a factor of 10. Such a decrease in computational costs is not significant enough given the efficiency of lumped-mass rockfall simulation codes, such as Rockyfor3D, in terms of computational duration. However, for more complex rockfall simulations approach based on an explicit modeling of rocks shapes (e.g., Leine et al. 2014) and inducing substantial computational costs, PEM-based approach may be of great interest. For any type of rockfall simulation code, the advantage of these approaches is much more pronounced when varying the type of distribution law, providing the range is the same. The PEM approach allows considering different distribution laws without additional rockfall simulations.

From an operational point of view, the results from rockfall trajectory simulations are often synthesized through quantiles of the rock passing height and kinetic energy distributions for designing protective structures, in particular. Though a major issue, the number of simulations required to get stable values of these quantiles is rarely addressed. In 
particular, getting a statistically relevant value of the $99 \%$ quantile requires much more simulations than the $95 \%$ quantile. For the reference case of this study, the results suggest that 1000 blocks are sufficient to obtain a relevant value for both the fence height and fence capacity when considering the $5 \%$ residual hazard quantile, i.e., corresponding to $95 \%$ of the blocks (Fig. 4). If the $1 \%$ residual hazard quantile is considered, the number of blocks should be 10,000 rocks to get a relevant fence height. This figure corresponds to the number of blocks released which, in this case, is very close to the number of blocks reaching the structure. In general, the criterion to consider should be the number of rock reaching the structure location. This quantity can be significantly smaller than the number of released rocks. Indeed, both the probabilities that a block stops on the slope before reaching the structure or deviate from the release point-structure axis have to be considered as they increase with the distance from the release point to the structure.

These considerations naturally lead to question the relevance of the few recommendations concerning the design of rockfall protection fences. The fence height is recommended to be based on the $95 \%$ block passing height quantile (ONR 2013; UNI11211-4 2012). In terms of risk reduction, this quantile may be considered insufficient. But fence height curves exhibit a significant increase from the $5 \%$ to the $1 \%$ FRH quantile with an increase in height of $60 \%$ for the reference case for example. This clearly shows that improving the efficiency of the fence with the aim of intercepting $99 \%$ of the blocks will significantly increases the cost of the fence and should be justified by a cost-benefit analysis. In addition, this height is sometimes proposed to be ponderated by a safety coefficient in the 1.05-1.3 range depending on the consequences of the structure failure (ONR 2013). The results presented in this study have shown that such a coefficient may allow accounting for the variability of the falling height and block volume except in the case of an error in the block volume, when considered deterministic (Fig. 7).

The passing height and rock translational kinetic energy of the block have been considered as fence design criterion. The choice of the location of the fence along the slope was not investigated in this research work although it is a crucial design parameter. The location of the fence has to be chosen to minimize fence height and energy according to the results from rocks trajectory simulations (Bourrier et al. 2009b) with consideration of other technical aspects, such as accessibility for example. In addition, the main limitation in the proposed approach is that the passing heights and energies were considered as independent random variables, which is not the case in general. Besides neither the impact point location (e.g., impact on the post or close to the supporting cables) nor the block trajectory inclination and rotational velocity were accounted for. A complete structure design would require accounting for the effective mechanical response of the structure considering the real block kinematics. Such an approach could be based on the coupling of a mechanical model of the fence together with trajectory simulations (Bourrier et al. 2015). Such a design is all the more complex and time-consuming in particular given the number of parameters managing the rock kinematics and their potential dependencies. As long as such designs are not conducted, designers should verify that simplifications made in the structure design are conservative.

\section{Conclusion}

Through an analysis on a well-documented study site, the presented research work allowed assessing the relevance of a methodology for the design of rockfall protection structures using rockfall propagation simulations. The choice of simulation parameters was also 
studied focusing on the parameters related to the release conditions-falling height and rock volume-which are much conditioned to expert-based assessment.

Following the proposed methodology for the study site, the assessment of the rock volume and assessment of its variation range were shown of major importance compared to the initial falling height and rock volume distribution law. This result tend to show that priority should be given to rock volume assessment during field works dedicated to rockfall simulation parameters assessment. In practice, this assessment relies on the monitoring of potentially instable rock volumes in the cliff as well as on the identification and characterization of the rocks observed along the slope in the site of interest. Direct analysis on the cliff requires technical and expensive measurements such as, for instance, the monitoring based on terrestrial laser scanning or photogrammetry. On the contrary, characterization of the observed rocks along the slope is an easy way of assessing the rock volume distribution. Besides, direct assessment on the cliff has to be completed by information on rocks potential fragmentation, while measurements along the slope generally implies the assumption that fragmentation mainly occurs at the beginning of rock propagation. This point emphasizes the importance of reliable information about rock fragmentation, while only few studies dedicated to this key point exist in the literature (Giacomini et al. 2009).

As regards the design of the protection fences, the study emphasized the importance of the choice of the target residual hazard on the structure characteristics in terms of both height and capacity. This choice is difficult since, in most countries, no clear reglementary rule exist. In addition, the fence design rarely integrates the use of mechanical models of the fence for a global assessment of the fence efficiency under variable impact conditions although such mechanical models were developed for most of the protection structures (Volkwein et al. 2011). Increased research dedicated to developing such design approaches (Bourrier et al. 2015) can be of major interest for defining target residual hazards values, related to the structure mechanical properties.

\section{Appendix 1: Quadrature rules}

We consider a random variable $Z$ (e.g., modeling a frequency), depending on an input random variable $Y$ (e.g., modeling a rock volume), such that $Z=f(Y)$ characterized by a mean $\mu_{Y}$, a standard deviation $\sigma_{Y}$ and a probability law. We also consider the function $T$ allowing the uniform or Gaussian standardization of $Y$, which, respectively, becomes $Y=$ $T(U)$ or $Y=T(X)$, with $U$ and $X$ uniform and standard Gaussian random variables. These standardizations are well known, see, for example, Rosenblatt (1952).

The mean $\mu_{Z}$ of $Z$ can be approximated using a quadrature rule, depending on its probability law and on the number of integration points $k$ (e.g., Zhou and Nowak 1988). In the case of a uniform standardization it becomes

$$
\mu_{Z}=\int_{0}^{1} f o T(u) p_{U}(u) d u \approx \hat{\mu}_{Z}=\sum_{j=1}^{k} f o T\left(u_{j}\right) \omega_{j}
$$

where $p_{U}$ is the uniform probability density function defined on $[0,1],\left(u_{j}, \omega_{j}\right)_{1 \leq j \leq k}$ are points and weights, given for $k=3,4,5$ in Table 4 .

In the case of a Gaussian standardization, the mean $\mu_{Z}$ becomes 
Table 4 Points and weights, uniform law with $\eta=\frac{\sqrt{245+14 \sqrt{70}}}{42}$ and $\xi=\frac{\sqrt{245-14 \sqrt{70}}}{42}$ and $\gamma=13 \sqrt{70}$

\begin{tabular}{llll}
\hline Points, weights & $k=3$ & $k=4$ & $k=5$ \\
\hline$u_{1}, \omega_{1}$ & $0.5-\frac{\sqrt{15}}{10}, 5 / 18$ & $0.5-\frac{\sqrt{525+70 \sqrt{30}}}{70}, \frac{18-\sqrt{30}}{72}$ & $0.5-\eta, \frac{322-\gamma}{1800}$ \\
$u_{2}, \omega_{2}$ & $0.5,4 / 9$ & $0.5-\frac{\sqrt{525-70 \sqrt{30}}}{70}, \frac{18+\sqrt{30}}{72}$ & $0.5-\xi, \frac{322+\gamma}{1800}$ \\
$u_{3}, \omega_{3}$ & $0.5+\frac{\sqrt{15}}{10}, 5 / 18$ & $0.5+\frac{\sqrt{525-70 \sqrt{30}}}{70}, \frac{18+\sqrt{30}}{72}$ & $0.5,128 / 450$ \\
$u_{4}, \omega_{4}$ & - & $0.5+\frac{\sqrt{525+70 \sqrt{30}}}{70}, \frac{18-\sqrt{30}}{72}$ & $0.5+\xi, \frac{322+\gamma}{1800}$ \\
$u_{5}, \omega_{5}$ & - & - & $0.5+\eta, \frac{322-\gamma}{1800}$ \\
\hline
\end{tabular}

Table 5 Points and weights, standard Gaussian law, for $k=$ $3,4,5$ integration points

\begin{tabular}{llll}
\hline Points, weights & $k=3$ & $k=4$ & $k=5$ \\
\hline$x_{1}, \omega_{1}$ & $-\frac{\sqrt{6}}{2}, 1 / 6$ & $-\sqrt{\frac{3+\sqrt{6}}{2}}, \frac{1}{4(3+\sqrt{6})}$ & $-2.0202,0.01126$ \\
$x_{2}, \omega_{2}$ & $0,2 / 3$ & $-\sqrt{\frac{3-\sqrt{6}}{2}}, \frac{1}{4(3-\sqrt{6})}$ & $-0.9586,0.2221$ \\
$x_{3}, \omega_{3}$ & $\frac{\sqrt{6}}{2}, 1 / 6$ & $\sqrt{\frac{3-\sqrt{6}}{2}}, \frac{1}{4(3-\sqrt{6})}$ & $0,0.5333$ \\
$x_{4}, \omega_{4}$ & - & $\sqrt{\frac{3+\sqrt{6}}{2}}, \frac{1}{4(3+\sqrt{6})}$ & $0.9586,0.2221$ \\
$x_{5}, \omega_{5}$ & - & - & $2.0202,0.01126$ \\
\hline
\end{tabular}

$$
\mu_{Z}=\int_{R} f o T(x) p_{X}(x) \mathrm{d} x \approx \hat{\mu}_{Z}=\sum_{j=1}^{k} f o T\left(x_{j}\right) \omega_{j}
$$

where $p_{X}$ is the Gaussian probability density function, $\left(x_{j}, \omega_{j}\right)_{1 \leq j \leq k}$ are points and weights, given for $k=3,4,5$ in Table 5. Baroth et al. (2007) or Daudon et al. (2013) showed that 4 and 5 points give already satisfying results.

\section{Appendix 2: Characterization of various distributions of rock volumes generated from 5 sets of rockfall simulations}

The effect of changing the distribution of rock volumes can be tested, considering uniform, Gaussian and beta distributions.

Uniform law is particularly useful if the variability of the input uncertain parameter is just characterized by bounds $\left[y_{\min }, y_{\max }\right]$. In this case, we define the random variable $Y_{1} \sim U\left(y_{\min }, y_{\max }\right)$ and points $y_{j}$ become, $\forall j=1, \ldots, k$

$$
y_{j}=y_{\min }+\left(y_{\max }-y_{\min }\right) \times u_{j}
$$

where $\left(u_{j}, \omega_{j}\right)_{1 \leq j \leq k}$ are given in Table $4(k=5)$.

The same values $\left(y_{2}, y_{3}, y_{4}\right)$ can be used to define a Gaussian random variable $Y_{2}$, with mean $\mu_{Y_{2}}$ and standard deviation $\sigma_{Y_{2}}$ such that 
Table 6 Characterization of laws tested from five calculations $\left(y_{j}\right)_{1 \leq j \leq 5}$

\begin{tabular}{llll}
\hline Variable & Loi & Table, column & definition of parameters \\
\hline$Y_{1}$ & $U\left(y_{\min }, y_{\max }\right)$ & $4, k=5$ & $\mu_{Y_{1}}=y_{3} ; C v_{Y_{1}}=\frac{y_{3}\left(1-u_{2}\right)-y_{2}}{\sqrt{3} y_{3}\left(1-2 u_{2}\right)}$ \\
$Y_{2}$ & $N\left(\mu_{Y_{2}}, \sigma_{Y_{2}}^{2}\right)$ & $5, k=3$ & $\mu_{Y_{2}}=y_{3} ; C v_{Y_{2}}=\frac{y_{2}-y_{3}}{x_{1} y_{3}}$ \\
$Y_{3}$ & $\operatorname{Beta}(\alpha, \beta)$ & $4, k=3$ & $\mu_{Y_{3}}=1.25 ; \sigma_{Y_{3}}=0.43$ if $(\alpha=\beta=1)$ \\
\hline
\end{tabular}

$$
y_{j}=\mu_{Y_{2}}+\sigma_{Y_{2}} \times x_{j}
$$

with $\left(x_{2}, x_{3}, x_{4}\right)$ are given in Table $5(k=3)$.

If $Y_{3}$ is beta-distributed with parameters $\alpha$ and $\beta$, i.e., $Y_{3} \sim \operatorname{Beta}(\alpha, \beta)$, we can also deduce relations between $\left(y_{1}, \ldots, y_{5}\right)$ and integration points (Table 6). Choosing arbitrarly a symmetric law, i.e., $\alpha=\beta$, such that

$$
\begin{gathered}
y_{j}=y_{\min }+\left(y_{\max }-y_{\text {min }}\right) \times \operatorname{Beta}(\alpha, \alpha) \\
y_{j}=\frac{u_{j}^{\alpha-1}\left(1-u_{j}\right)^{\beta-1}}{\int_{0}^{1} t^{\alpha-1}(1-t)^{\beta-1} \mathrm{~d} t}
\end{gathered}
$$

From Table 6, standard deviations are deduced such that : $\sigma_{Y_{j}}=C v_{Y_{j}} \mu_{Y_{j}}, j=1, \ldots, k$.

\section{References}

Abramowitz M, Stegun I (1970) Handbook of mathematical functions. Dower Publications Inc, New York Agliardi F, Crosta GB, Frattini P (2009) Integrating rockfall risk assessment and countermeasure design by 3D modelling techniques. Nat Hazards Earth Syst Sci 9(4):1059-1073

Baroth J, Bressolette P, Chauvière C, Fogli M (2007) An efficient SFE method using Lagrange Polynomials: application to nonlinear mechanical problems with uncertain parameters. Compute Methods Appl Mech Eng 196(45-48):4419-4429

Bourrier F, Dorren L, Nicot F, Berger F, Darve F (2009a) Toward objective rockfall trajectory simulation using a stochastic impact model. Geomorphology 110:68-79

Bourrier F, Eckert N, Nicot F, Darve F (2009b) Bayesian stochastic modeling of a spherical rock bouncing on a coarse soil. Nat Hazards Earth Syst Sci 9(3):831-846

Bourrier F, Lambert S, Baroth J (2015) A reliability-based approach for the design of rockfall protection fences. Rock Mech Rock Eng 48:247-259

Christchurch City Council (2013) Technical guideline for rockfall protection structure. Christchurch City Council, Christchurch

Corominas J, Copons R, Moya J, Vilaplana JM, Altimir J, Amigo J (2005) Quantitative assessment of the residual risk in a rockfall protected area. Landslides 2(4):343-357

Daudon D, Baroth J, Ma Y, Perrotin P, Mommessin M (2013) Sensitivity of a reinforced concrete protective gallery under a snow avalanche load. Struct Saf 41:47-56

Dorren LK, Berger A, Putters F (2006) Real size experiments and 3D simulation of rockfall on forested and non-forested slopes. Nat Hazards Earth Syst Sci 6:145-153

Eckert N, Parent E, Faug T, Naaim M (2008) Optimal design under uncertainty of a passive defense structure against snow avalanches: from a general Bayesian framework to a simple analytical model. Nat Hazards Earth Syst Sci 8(5):1067-1081

EOTA (2013) ETAG 027: Guideline for European approval of rockfall protection kits, European organisation for technical approvals, Brussels, Belgium

Ferrero AM, Migliazza M, Roncella R, Segalini A (2011) Rock cliffs hazard analysis based on remote geostructural surveys: the Campione del Garda case study (Lake Garda, Northern Italy). Geomorphology 125:457-471 
Giacomini A, Buzzi O, Renard B, Giani GP (2009) Experimental studies on fragmentation of rock falls on impact with rock surfaces. Int J Rock Mech Min Sci 46(4):708-715

Guzzetti F, Reichenbach P, Ghigi S (2004) Rockfall hazard and risk assessment along a transportation corridor in the Nera valley, central Italy. Environ Manag 34(2):191-208

Hantz D (2012) Quantitative assessment of diffuse rock fall hazard along a cliff foot. Nat Hazards Earth Syst Sci 11:1303-1309

Lambert S, Bourrier F (2013) Design of rockfall protection embankments: a review. Eng Geol 154:77-88

Lambert S, Bourrier F, Toe D (2013) Improving three-dimensional rockfall trajectory simulation codes for assessing the efficiency of protective embankments. Int J Rock Mech Min Sci 60:26-36

Leine RI, Schweizer A, Christen M, Glover J, Bartelt P, Gerber W (2014) Simulation of rockfall trajectories with consideration of rock shape. Multibody Syst Dyn 32(2):241-271

Mavrouli O, Corominas J (2010) Rockfall vulnerability assessment for reinforced concrete buildings. Nat Hazards Earth Syst Sci 10(10):2055-2066

Millard A, Gosse G, Heinfung G, Defaux G, Mohamed A (2000) Reliability analysis of a reinforced concrete cooling tower. In: ASS IACM 2000, fourth international colloquium on computation of shell and spatial structures. Chania, Crete, Greece

Mignelli C, Lo Russo S, Peila D (2012) ROckfall risk MAnagement assessment: the RO.MA. approach. Nat Hazards 62(3):1109-1123

ONR (2013) NORM 24810: Technical protection against rockfall-terms and definitions, effects of actions, design, monitoring and maintenance. Austrian standard institute, Vienna

Peila D, Ronco C (2009) Design of rockfall net fences and the new ETAG 027 European guideline. Nat Hazards Earth Syst Sci 9:1291-1298

Rosenblatt M (1952) Remarks on a multiformation. Ann Math Stat 23:470-472

Schweiger HF (2001) Reliability analysis in geotechnics with deterministic finite elements. Int J Geomech $1(4): 389-413$

UNI11211-4 (2012) Rockfall protective measures. Part 4: definitive and executive design. Ente Nazionale Italiano di Unificazione, Milano

Volkwein A, Schellenberg K, Labiouse V, Agliardi F, Berger F, Bourrier F, Dorren LKA, Gerber W, Jaboyedoff M (2011) Rockfall characterisation and structural protection-a review. Nat Hazards Earth Syst Sci 11(9):2617-2651

Zhou J, Nowak AS (1988) Integration formulas to evaluate functions of random variables. Struct Saf 5(4):267-284 